\title{
Extended Tensor Products and Generalization of the Notion of Entanglement
}

\author{
Andrei Khrennikov \\ International Center for Mathematical Modelling \\ in Physics and Cognitive Sciences \\ Linnaeus University, Växjö, Sweden \\ Elemer E. Rosinger \\ Department of Mathematics, University of Pretoria \\ South-African Republic
}

October 8, 2018

\begin{abstract}
Motivated by the novel applications of the mathematical formalism of quantum theory and its generalizations in cognitive science, psychology, social and political sciences, and economics, we extend the notion of the tensor product and entanglement. We also study the relation between conventional entanglement of complex qubits and our generalized entanglement. Our construction can also be used to describe entanglement in the framework of non-Archimedean physics. It is also possible to construct tensor products of non-Archimedean (e.g., $p$-adic) and complex Hilbert spaces.
\end{abstract}

\section{Introduction}

The notion of the tensor product plays a crucial role in quantum theory: states of composite quantum systems belong to the tensor product of Hilbert state spaces of subsystems. The tensor product is the basic structure of quantum information theory; in particular, such a fundamental notion as 
entanglement is defined on the basis of the tensor product. Recently the quantum formalism and its generalization started to be applied outside of the domain of quantum physics: in cognitive science, psychology, economics, and finances, see, e.g., [1], 2] and bibliography in these works. In these applications it became clear that the conventional quantum formalism is too special and restricted to cover all these novel applications. More general quantum-like models describing nonclassical flows of information are on demand. In particular, composite cognitive and social systems need not be described by the conventional tensor product; they may exhibit other types of entanglement which are not reduced to the conventional quantum entanglement. This was one of motivations for E. Rosinger to extend the notion of the conventional tensor product [3] and entanglement. In this note we continue the activity in this direction. The tensor construction of [3] is extened even further to obtain a possibility to use operator algebras, instead of fields of scalars. We consider a few examples related to generalized tensor representation of the standard Bell states. Among other possible applications of the extended tensor product we can mention the description of generalized quantum systems which state spaces are not simply complex Hilbert spaces, but (in general noncommutative) groups. We can also describe entanglement in the framework of non-Archimedean physics, e.g., 4], [5]. It is also possible to construct tensor products of non-Archimedean (e.g., p-adic) and complex Hilbert spaces. The latter is a subject of our further investigations.

\section{Definition of extended tensor products}

Let

$\alpha: X \times X \longrightarrow X, \beta: Y \times Y \longrightarrow Y$

be two arbitrary maps.

Let us denote by $Z$ the set of all finite sequences of pairs

$$
\left(x_{1}, y_{1}\right), \ldots,\left(x_{n}, y_{n}\right)
$$

where $n \in \mathbf{N}_{1}$, while $x_{i} \in X, y_{i} \in Y$, with $1 \leq i \leq n$.

We define on $Z$ the binary operation $\gamma$ simply by the concatenation of 
the sequences (1). It follows that $\gamma$ is associative, therefore, each sequence (4) can be written as

$$
\left(x_{1}, y_{1}\right), \ldots,\left(x_{n}, y_{n}\right)=\left(x_{1}, y_{1}\right) \gamma\left(x_{2}, y_{2}\right) \gamma \ldots \gamma\left(x_{n}, y_{n}\right)
$$

where for $n=1$, the right hand term is understood to be simply $\left(x_{1}, y_{1}\right)$. Obviously, if $X$ or $Y$ have at least two elements, then $\gamma$ is not commutative.

Then (1), (2) give

$$
Z=\left\{\begin{array}{l|l}
\left(x_{1}, y_{1}\right) \gamma\left(x_{2}, y_{2}\right) \gamma \ldots \gamma\left(x_{n}, y_{n}\right) & \begin{array}{l}
n \geq 1 \\
x_{i} \in X, y_{i} \in Y, 1 \leq i \leq n
\end{array}
\end{array}\right\}
$$

which obviously results in

$$
X \times Y \subseteq Z
$$

Now we define on $Z$ an equivalence relation $\approx_{\alpha, \beta}$ as follows. Two sequences in (1) are equivalent, if and only if they are identical, or each can be obtained from the other by a finite number of applications of the following operations :

(5) permuting the pairs $\left(x_{i}, y_{i}\right)$ within the sequence

(6) replacing $\left(\alpha\left(x_{1}, x_{1}^{\prime}\right), y_{1}\right),\left(x_{2}, y_{2}\right), \ldots,\left(x_{n}, y_{n}\right)$ with $\left(x_{1}, y_{1}\right),\left(x_{1}^{\prime}, y_{1}\right),\left(x_{2}, y_{2}\right), \ldots,\left(x_{n}, y_{n}\right)$, or vice-versa

(7) replacing $\left(x_{1}, \beta\left(y_{1}, y_{1}^{\prime}\right)\right),\left(x_{2}, y_{2}\right), \ldots,\left(x_{n}, y_{n}\right)$ with $\left(x_{1}, y_{1}\right),\left(x_{1}, y_{1}^{\prime}\right),\left(x_{2}, y_{2}\right), \ldots,\left(x_{n}, y_{n}\right)$, or vice-versa

Let us note that, in view of the rather general related result in Lemma 1. below, the binary relation $\approx_{\alpha, \beta}$ defined above on $Z$ is indeed an equivalence relation.

Finally, the tensor product of $(X, \alpha)$ and $(Y, \beta)$ is defined to be the quotient space 


$$
X \otimes_{\alpha, \beta} Y=Z / \approx_{\alpha, \beta}
$$

with the mapping $\tau_{\alpha, \beta}$ induced through the inclusion (4) by the canonical quotient embedding corresponding to (8), namely

$$
X \times Y \ni(x, y) \stackrel{\tau_{\alpha, \beta}}{\longmapsto} x \bigotimes_{\alpha, \beta} y \in X \bigotimes_{\alpha, \beta} Y
$$

where as in the usual case of tensor products, we denote by $x \bigotimes_{\alpha, \beta} y$, or simply $x \otimes y$, the $\approx_{\alpha, \beta}$ equivalence class of $(x, y)$.

Furthermore, the equivalence $\approx_{\alpha, \beta}$ is compatible with the semigroup structure $(Z, \gamma)$, thus $(8)$ has in fact the stronger form which gives a commutative semigroup structure on the resulting generalized tensor product $X \bigotimes_{\alpha, \beta} Y$, namely

$$
\left(X \bigotimes_{\alpha, \beta} Y, \gamma / \approx_{\alpha, \beta}\right)=(Z, \gamma) / \approx_{\alpha, \beta}
$$

For simplicity, however, we shall write $\gamma$ instead of $\gamma / \approx_{\alpha, \beta}$.

In this way, the elements of $X \bigotimes_{\alpha, \beta} Y$ are all the expressions

$$
x_{1} \bigotimes_{\alpha, \beta} y_{1} \gamma x_{2} \bigotimes_{\alpha, \beta} y_{2} \gamma \ldots \gamma x_{n} \bigotimes_{\alpha, \beta} y_{n}
$$

with $n \geq 1$ and $x_{i} \in X, y_{i} \in Y$, for $1 \leq i \leq n$.

\section{How large are the extended tensor prod- ucts?}

Before going further, let us see when is the mapping (9) injective. A necessary condition is given by

\section{Proposition 1.}


If the mapping $\tau_{\alpha, \beta}$ in (9), namely

$$
X \times Y \ni(x, y) \stackrel{\tau_{\alpha, \beta}}{\longmapsto} x \bigotimes_{\alpha, \beta} y \in X \bigotimes_{\alpha, \beta} Y
$$

is injective, then the binary operations $\alpha$ and $\beta$ are associative.

\section{Proof.}

We first show that

$$
\alpha \text { not associative } \Longrightarrow \tau_{\alpha, \beta} \text { not injective }
$$

Indeed, let $a, b, c \in X$, such that $d=\alpha(\alpha(a, b), c) \neq \alpha(a, \alpha(b, c))=e$. Further, let $y \in X$. Then in view of (6), we have

$$
\begin{aligned}
(d, y) & =(\alpha(\alpha(a, b)), c), y) \approx_{\alpha, \beta}(\alpha(a, b), y) \gamma(c, y) \approx_{\alpha, \beta} \\
& \approx_{\alpha, \beta}(a, y) \gamma(b, y) \gamma(c, y) \\
& \approx_{\alpha, \beta}(a, y) \gamma(\alpha(b, c), y) \approx_{\alpha, \beta} \\
& \approx_{\alpha, \beta}(\alpha(a, \alpha(b, c)), y)=(e, y)
\end{aligned}
$$

hence $(d, y) \approx_{\alpha, \beta}(e, y)$, while obviously $(d, y) \neq(e, y)$.

In a similar manner, we also have

$$
\beta \text { not associative } \Longrightarrow \tau_{\alpha, \beta} \text { not injective }
$$

The converse of Proposition 3.1. does not hold, as illustrated in

\section{Example 1.}

The above definition contains as a particular case the usual tensor products of groups. And for Abelian groups one has

$$
\mathbf{Z} /(m) \bigotimes_{\mathbf{Z}} \mathbf{Z} /(n)=\mathbf{Z} /(d)
$$


for $m, n \in \mathbf{N}$, and $d$ the greatest common divisor of $m$ and $n$. Thus in particular

$$
\mathbf{Z} /(2) \otimes_{\mathbf{Z}} \mathbf{Z} /(3)=0
$$

Clearly, the binary operation $\gamma$ on $Z$ will canonically lead by this quotient operation to a commutative and associative binary operation on $X \otimes_{\alpha, \beta} Y$, which for convenience is denoted by the same $\gamma$, although this time it depends on $\alpha$ and $\beta$.

The customary and highly particular situation is when $X$ and $Y$ are semigroups, groups, or even vector spaces over some field $\mathbf{K}$. In this case $\alpha, \beta$ and $\gamma$ are as usual denoted by + , that is, the sign of addition.

It is easy to note that in the construction of tensor products above, it is not necessary for $(X, \alpha)$ and $(Y, \beta)$ to be semigroups, let alone groups, or for that matter, vector spaces. Indeed, it is sufficient that $\alpha$ and $\beta$ are arbitrary binary operations on $X$ and $Y$, respectively.

Also, as seen above, $\alpha$ and $\beta$ need not be commutative either. However, the tensor product $X \otimes_{\alpha, \beta} Y$, with the respective binary operation $\gamma$, will nevertheless be commutative and associative.

It is important to note that the tensor products defined above have a universality property which is a natural generalization of the corresponding well known one for usual tensor products.

\section{Extended concepts of entanglement}

\section{Definition 1.}

Given two binary operations $\alpha: X \times X \longrightarrow X$ and $\beta: Y \times Y \longrightarrow Y$. An element $w \in X \bigotimes_{\alpha, \beta} Y$ is called entangled, if and only if it is not of the form

$$
w=x \bigotimes_{\alpha, \beta} y
$$


for some $x \in X$ and $y \in Y$.

\section{Note 1.}

1) Since it was noted that the usual tensor products are particular cases of the tensor products defined in this section, it follows that the definition of entanglement given above does indeed generalize the usual concept of entanglement.

2) It is important to note that generalized tensor products (8) can have an interest even when the corresponding mappings (9) are not injective. Indeed, if for instance in such cases one still has the strict inclusion

$$
\tau_{\alpha, \beta}(X \times Y) \subset X \bigotimes_{\alpha, \beta} Y, \tau_{\alpha, \beta}(X \times Y) \neq X \bigotimes_{\alpha, \beta} Y
$$

then there are still entangled elements in $X \bigotimes_{\alpha, \beta} Y$, namely, those in the nonvoid set

$$
X \bigotimes_{\alpha, \beta} Y \backslash \tau_{\alpha, \beta}(X \times Y)
$$

3) As seen in elsewhere, tensor products can be defined in far more general ways than above. And with such far more general definitions there are plenty of cases when the mappings corresponding to (9) will be injective.

In the construction of tensor products above, we used the following easy to prove

\section{Lemma 1.}

Let on a nonvoid set $E$ be given a family $\left(\equiv_{i}\right)_{i \in I}$ of symmetric binary relations. Further, let us define on $E$ the binary relation $\approx$ as follows. For $a, b \in E$, we have $a \approx b$, if and only if $a=b$, or there exists a finite sequence

$$
a=c_{0} \equiv_{i_{0}} c_{1} \equiv_{i_{1}} c_{2} \equiv_{i_{2}} \ldots \equiv_{i_{n-2}} c_{n-1} \equiv_{i_{n-1}} c_{n}=y
$$

where $c_{1}, \ldots, c_{n-1} \in E$.

Then $\approx$ is an equivalence relation on $E$. 


\section{Further ways to extend the concepts of ten- sor products and entanglements}

Let

$\mathcal{A} \subseteq X^{X}, \mathcal{B} \subseteq Y^{Y}$

be arbitrary mappings. Then we define

$\mathcal{C} \subseteq Z^{Z}$

as the set of all pairs of mappings

$C=(A, B) \in \mathcal{A} \times \mathcal{B}$

which act according to

$$
\begin{aligned}
Z \ni\left(x_{1}, y_{1}\right), \ldots,\left(x_{n}, y_{n}\right) & \longmapsto \\
& \longmapsto\left(A\left(x_{1}\right), B\left(y_{1}\right)\right), \ldots,\left(A\left(x_{n}\right), B\left(y_{n}\right)\right) \in Z
\end{aligned}
$$

Now we consider the particular case when

$$
X=Y, \mathcal{A}=\mathcal{B}
$$

and then we define on $Z$ the equivalence relation $\approx_{\mathcal{A}}$ as follows. Two sequences in (1) are equivalent, if and only if they are identical, or each can be obtained from the other by a finite number of applications of the following operations :

(19) permuting the pairs $\left(x_{i}, y_{i}\right)$ within the sequence

(20) replacing $\left(A\left(x_{1}\right), B\left(y_{1}\right)\right), \ldots,\left(A\left(x_{n}\right), B\left(y_{n}\right)\right)$ with $\left(B A\left(x_{1}\right), y_{1}\right), \ldots,\left(B A\left(x_{n}\right), y_{n}\right)$, or with 
$\left(x_{1}, A B\left(y_{1}\right)\right), \ldots,\left(x_{n}, A B\left(y_{n}\right)\right)$, or with

$(A, B)\left(\left(x_{1}, y_{1}\right), \ldots,\left(x_{n}, y_{n}\right)\right)$, or vice-versa,

where $A, B \in \mathcal{A}$

In view of the above Lemma 1 ., it follows that $\approx_{\mathcal{A}}$ is indeed an equivalence relation on $Z$, therefore, we can define the tensor product

$$
X \otimes_{\mathcal{A}} X=Z / \approx_{\mathcal{A}}
$$

with the mapping $\tau_{\mathcal{A}}$ induced through the inclusion (4) by the canonical quotient embedding corresponding to (21), namely

$$
X \times X \ni(x, y) \stackrel{\tau_{\mathcal{A}}}{\longmapsto} x \bigotimes_{\mathcal{A}} y \in X \bigotimes_{\mathcal{A}} Y
$$

where as in the usual case of tensor products, we denote by $x \bigotimes_{\mathcal{A}} y$, or simply $x \otimes y$, the $\approx_{\mathcal{A}}$ equivalence class of $(x, y)$.

Furthermore, the equivalence $\approx_{\mathcal{A}}$ is compatible with the semigroup structure $(Z, \gamma)$, thus $(21)$ has in fact the stronger form which gives a commutative semigroup structure on the resulting generalized tensor product $X \bigotimes_{\mathcal{A}} X$, namely

$$
\left(X \bigotimes_{\mathcal{A}} X, \gamma / \approx_{\mathcal{A}}\right)=(Z, \gamma) / \approx_{\mathcal{A}}
$$

For simplicity, however, we shall write $\gamma$ instead of $\gamma / \approx_{\mathcal{A}}$.

In this way, the elements of $X \otimes_{\mathcal{A}} X$ are all the expressions

$$
x_{1} \bigotimes_{\mathcal{A}} y_{1} \gamma x_{2} \bigotimes_{\mathcal{A}} y_{2} \gamma \ldots \gamma x_{n} \bigotimes_{\mathcal{A}} y_{n}
$$

with $n \geq 1$ and $x_{i}, y_{i} \in X$, for $1 \leq i \leq n$. 


\section{Further extensions of the concept of entan- glement}

The above tensor products

$X \bigotimes_{\mathcal{A}} X$ and $X \bigotimes_{\alpha, \mathcal{A}} X$

have corresponding concepts of entanglement according to obvious extensions of Definition 1. above.

\section{A Mixture of the Above}

Let

$\alpha: X \times X \longrightarrow X$

and

$\mathcal{A} \subseteq X^{X}$

and consider

$\mathcal{C} \subseteq Z^{Z}$

as the set of all pairs of mappings

$C=(A, B) \in \mathcal{A} \times \mathcal{A}$

which act according to

$$
\begin{aligned}
Z \ni\left(x_{1}, y_{1}\right), \ldots,\left(x_{n}, y_{n}\right) & \longmapsto \\
& \longmapsto\left(A\left(x_{1}\right), B\left(y_{1}\right)\right), \ldots,\left(A\left(x_{n}\right), B\left(y_{n}\right)\right) \in Z
\end{aligned}
$$

Define now on $Z$ the equivalence relation $\approx_{\alpha, \mathcal{A}}$ as follows. Two sequences in (1) are equivalent, if and only if they are identical, or each can be obtained 
from the other by a finite number of applications of the following operations :

(25) permuting the pairs $\left(x_{i}, y_{i}\right)$ within the sequence

(26) replacing $\left(\alpha\left(x_{1}, x_{1}^{\prime}\right), y_{1}\right),\left(x_{2}, y_{2}\right), \ldots,\left(x_{n}, y_{n}\right)$ with

$\left(x_{1}, y_{1}\right),\left(x_{1}^{\prime}, y_{1}\right),\left(x_{2}, y_{2}\right), \ldots,\left(x_{n}, y_{n}\right)$, or vice-versa

(27) replacing $\left(x_{1}, \alpha\left(y_{1}, y_{1}^{\prime}\right)\right),\left(x_{2}, y_{2}\right), \ldots,\left(x_{n}, y_{n}\right)$ with

$\left(x_{1}, y_{1}\right),\left(x_{1}, y_{1}^{\prime}\right),\left(x_{2}, y_{2}\right), \ldots,\left(x_{n}, y_{n}\right)$, or vice-versa

(28) replacing $\left(A\left(x_{1}\right), B\left(y_{1}\right)\right), \ldots,\left(A\left(x_{n}\right), B\left(y_{n}\right)\right)$ with

$\left(B A\left(x_{1}\right), y_{1}\right), \ldots,\left(B A\left(x_{n}\right), y_{n}\right)$, or with

$\left(x_{1}, A B\left(y_{1}\right)\right), \ldots,\left(x_{n}, A B\left(y_{n}\right)\right)$, or with

$(A, B)\left(\left(x_{1}, y_{1}\right), \ldots,\left(x_{n}, y_{n}\right)\right)$, or vice-versa,

where $A, B \in \mathcal{A}$

Then in view of Lemma 1 . above, it follows that $\approx_{\alpha, \mathcal{A}}$ is indeed an equivalence relation on $Z$, therefore, we can define the tensor product

$$
X \bigotimes_{\alpha, \mathcal{A}} X=Z / \approx_{\alpha, \mathcal{A}}
$$

with the mapping $\tau_{\mathcal{A}}$ induced through the inclusion (4) by the canonical quotient embedding corresponding to (31), namely

$$
X \times X \ni(x, y) \stackrel{\tau_{\alpha, \mathcal{A}}}{\longmapsto} x \bigotimes_{\alpha, \mathcal{A}} y \in X \bigotimes_{\alpha, \mathcal{A}} Y
$$

where as in the usual case of tensor products, we denote by $x \bigotimes_{\alpha, \mathcal{A}} y$, or simply $x \otimes y$, the $\approx_{\alpha, \mathcal{A}}$ equivalence class of $(x, y)$.

Furthermore, the equivalence $\approx_{\alpha, \mathcal{A}}$ is compatible with the semigroup structure $(Z, \gamma)$, thus $(29)$ has in fact the stronger form which gives a commutative semigroup structure on the resulting generalized tensor product $X \bigotimes_{\alpha, \mathcal{A}} X$, namely

$$
\left(X \bigotimes_{\alpha, \mathcal{A}} X, \gamma / \approx_{\alpha, \mathcal{A}}\right)=(Z, \gamma) / \approx_{\alpha, \mathcal{A}}
$$

For simplicity, however, we shall write $\gamma$ instead of $\gamma / \approx_{\alpha, \mathcal{A}}$. 
In this way, the elements of $X \bigotimes_{\alpha, \mathcal{A}} X$ are all the expressions

$$
x_{1} \bigotimes_{\alpha, \mathcal{A}} y_{1} \gamma x_{2} \bigotimes_{\alpha, \mathcal{A}} y_{2} \gamma \ldots \gamma x_{n} \bigotimes_{\alpha, \mathcal{A}} y_{n}
$$

with $n \geq 1$ and $x_{i}, y_{i} \in X$, for $1 \leq i \leq n$.

\section{Relationship between the three tensor prod- ucts}

$X \bigotimes_{\alpha, \alpha} X, \quad X \bigotimes_{\mathcal{A}} X$ and $X \bigotimes_{\alpha, \mathcal{A}} X$

In view of Lemma 2 . below, we have the surjective mapping, see (11)

$$
\begin{aligned}
& \lambda_{\alpha, \mathcal{A}}: X \bigotimes_{\alpha, \alpha} X \ni x_{1} \bigotimes_{\alpha, \alpha} y_{1} \gamma x_{2} \bigotimes_{\alpha, \alpha} y_{2} \gamma \ldots \gamma x_{n} \bigotimes_{\alpha, \alpha} y_{n} \longmapsto \\
& \longmapsto x_{1} \bigotimes_{\alpha, \mathcal{A}} y_{1} \gamma x_{2} \bigotimes_{\alpha, \mathcal{A}} y_{2} \gamma \ldots \gamma x_{n} \bigotimes_{\alpha, \mathcal{A}} y_{n} \in X \bigotimes_{\alpha, \mathcal{A}} X
\end{aligned}
$$

as well as the surjective mapping, see (24)

$$
\begin{aligned}
& \mu_{\alpha, \mathcal{A}}: X \bigotimes_{\mathcal{A}} X \ni x_{1} \bigotimes_{\mathcal{A}} y_{1} \gamma x_{2} \bigotimes_{\mathcal{A}} y_{2} \gamma \ldots \gamma x_{n} \bigotimes_{\mathcal{A}} y_{n} \longmapsto \\
& \longmapsto x_{1} \bigotimes_{\alpha, \mathcal{A}} y_{1} \gamma x_{2} \bigotimes_{\alpha, \mathcal{A}} y_{2} \gamma \ldots \gamma x_{n} \bigotimes_{\alpha, \mathcal{A}} y_{n} \in X \bigotimes_{\alpha, \mathcal{A}} X
\end{aligned}
$$

\section{A Further Lemma}

\section{Lemma 2.}

Let on a nonvoid set $E$ be given two families $\left(\equiv_{i}\right)_{i \in I}$ and $\left(\equiv_{j}\right)_{j \in J}$ of symmetric binary relations, where $I \subseteq J$. Let us denote by $\approx_{I}$ and $\approx_{j}$ the respective equivalence relations in $E$ constructed according to Lemma 1. Then for $a, b \in E$, we have

$$
a \approx_{I} b \Longrightarrow a \approx_{J} b
$$


therefore, we have the surjective mapping

$$
E / \approx_{I} \ni(a)_{\approx_{I}} \longmapsto(a)_{\approx_{J}} \in E / \approx_{J}
$$

where $(a)_{\approx_{I}}$ and $(a)_{\approx_{J}}$ are the equivalence classes of $a \in E$ with respect to the corresponding equivalence relations $\approx_{I}$ and $\approx_{J}$.

\section{Note 2.}

The above relation (20) means that by enlarging the family $\left(\equiv_{i}\right)_{i \in I}$ of symmetric binary relations on a set $E$, one decreases the corresponding quotient space $E / \approx_{I}$.

Examples of generalized tensor product representations of the conventional Bell's states:

Let $X=Y=H=\mathbf{C}^{2}, \alpha=\beta=+$ on $H, \mathcal{A}=\mathcal{B}=L(H)$ the algebra of linear operators on the Hilbert space $H$

We recall the Pauli matrices

$\sigma_{x}=\left(\begin{array}{cc}0 & 1 \\ 1 & 0\end{array}\right) \quad \sigma_{y}=\left(\begin{array}{cc}0 & -i \\ i & 0\end{array}\right) \quad \sigma_{z}=\left(\begin{array}{cc}1 & 0 \\ 0 & -1\end{array}\right)$

and the Hadamard matrix

$H=(1 / \sqrt{2})\left(\begin{array}{cc}1 & 1 \\ 1 & -1\end{array}\right)$

which satisfy

$$
\begin{aligned}
& \sigma_{x}^{2}=\sigma_{y}^{2}=\sigma_{z}^{2}=-i \sigma_{x} \sigma_{y} \sigma_{z}=H^{2}=I=\left(\begin{array}{ll}
1 & 0 \\
0 & 1
\end{array}\right) \\
& \sigma_{x} \sigma_{y}=\left(\begin{array}{cc}
i & 0 \\
0 & -i
\end{array}\right)=i \sigma_{z}, \quad \sigma_{y} \sigma_{x}=\left(\begin{array}{cc}
-i & 0 \\
0 & i
\end{array}\right)=-i \sigma_{z} \\
& \sigma_{x} \sigma_{z}=\left(\begin{array}{cc}
0 & -1 \\
1 & 0
\end{array}\right)=i \sigma_{y}, \quad \sigma_{z} \sigma_{x}=\left(\begin{array}{cc}
0 & 1 \\
-1 & 0
\end{array}\right)=-i \sigma_{y}
\end{aligned}
$$




$$
\sigma_{y} \sigma_{z}=\left(\begin{array}{cc}
0 & i \\
i & 0
\end{array}\right)=i \sigma_{x}, \quad \sigma_{z} \sigma_{y}=\left(\begin{array}{cc}
0 & -i \\
-i & 0
\end{array}\right)=-i \sigma_{x}
$$

Further, we have

$$
\begin{aligned}
& \sigma_{x}(\mid 0>)=\left|1>, \quad \sigma_{x}(\mid 1>)=\right| 0> \\
& \sigma_{y}(\mid 0>)=i\left|1>, \quad \sigma_{y}(\mid 1>)=-i\right| 0> \\
& \sigma_{z}(\mid 0>)=\left|0>, \quad \sigma_{z}(\mid 1>)=-\right| 1> \\
& H(\mid 0>)=(|0>+| 1>) / \sqrt{2}, \quad H(\mid 1>)=(|0>-| 1>) / \sqrt{2}
\end{aligned}
$$

We take $\alpha=+$, thus the usual tensor product $\bigotimes$ is in fact $\bigotimes_{+,+}$.

1) Let us now take a Bell state

$$
\begin{aligned}
& \mid \psi>=(|1>| 0>+|0>| 1>) / \sqrt{2}= \\
& =(|1>\otimes| 0>+|0>\otimes| 1>) / \sqrt{2}= \\
& =\left(\left|1>\otimes_{+,+}\right| 0>+\left|0>\otimes_{+,+}\right| 1>\right) / \sqrt{2} \in H \otimes_{+,+} H=H \otimes H
\end{aligned}
$$

and apply to it the mapping $\lambda_{\alpha, \mathcal{A}}$ in (33) for various choices of the operators $A, B \in \mathcal{A}=\left\{\sigma_{x}, \sigma_{y}, \sigma_{z}, H\right\}$.

1.1) For $A=B=\sigma_{x}$, thus with $\mathcal{A}=\left\{\sigma_{x}\right\}$, we have in $H \bigotimes_{+, A} H$

$$
\begin{aligned}
& \lambda_{\alpha, \mathcal{A}}(\mid \psi>)=\left(\sigma_{x}, \sigma_{x}\right)(\mid \psi>)= \\
= & {\left[\left(\sigma_{x}(\mid 1>)\right)\left(\sigma_{x}(\mid 0>)\right)+\left(\sigma_{x}(\mid 0>)\right)\left(\sigma_{x}(\mid 1>)\right)\right] / \sqrt{2}=} \\
= & {[\mid 0>) \mid 1>)+\mid 1>) \mid 0>] / \sqrt{2}=\mid \psi>}
\end{aligned}
$$

1.2) For $A=B=\sigma_{y}$, thus with $\mathcal{A}=\left\{\sigma_{y}\right\}$, we have in $H \bigotimes_{+, A} H$ 


$$
\begin{aligned}
& \lambda_{\alpha, \mathcal{A}}(\mid \psi>)=\left(\sigma_{y}, \sigma_{y}\right)(\mid \psi>)= \\
= & {\left[\left(\sigma_{y}(\mid 1>)\right)\left(\sigma_{y}(\mid 0>)\right)+\left(\sigma_{y}(\mid 0>)\right)\left(\sigma_{y}(\mid 1>)\right)\right] / \sqrt{2}=} \\
= & {[(-i \mid 0>)(i \mid 1>))+(i \mid 1>)(-i \mid 0>)] / \sqrt{2}=\mid \psi>}
\end{aligned}
$$

1.3) For $A=B=\sigma_{z}$, thus with $\mathcal{A}=\left\{\sigma_{z}\right\}$, we have in $H \bigotimes_{+, A} H$

$$
\begin{aligned}
& \lambda_{\alpha, \mathcal{A}}(\mid \psi>)=\left(\sigma_{z}, \sigma_{z}\right)(\mid \psi>)= \\
= & {\left[\left(\sigma_{z}(\mid 1>)\right)\left(\sigma_{z}(\mid 0>)\right)+\left(\sigma_{z}(\mid 0>)\right)\left(\sigma_{z}(\mid 1>)\right)\right] / \sqrt{2}=} \\
= & {[-\mid 1>) \mid 0>)-\mid 0>) \mid 0>)] / \sqrt{2}=-\mid \psi>}
\end{aligned}
$$

1.4) For $A=\sigma_{x}, B=\sigma_{y}$, thus with $\mathcal{A}=\left\{\sigma_{x}, \sigma_{y}\right\}$, we have in $H \bigotimes_{+, A} H$$$
\lambda_{\alpha, \mathcal{A}}(\mid \psi>)=\left(\sigma_{x}, \sigma_{y}\right)(\mid \psi>)=
$$$$
=\left[\left(\sigma_{x}(\mid 1>)\right)\left(\sigma_{y}(\mid 0>)\right)+\left(\sigma_{x}(\mid 0>)\right)\left(\sigma_{y}(\mid 1>)\right)\right] / \sqrt{2}=
$$$$
=[\mid 0>)(i \mid 1>))-\mid 1>(i \mid 0>))] / \sqrt{2}=i \mid \chi>
$$

where

$$
|\chi>=(\mid 0>)| 1>)-|1>| 0>) / \sqrt{2}
$$

is a Bell state.

1.5) For $A=\sigma_{x}, B=\sigma_{z}$, thus with $\mathcal{A}=\left\{\sigma_{x}, \sigma_{z}\right\}$, we have in $H \bigotimes_{+, A} H$

$$
\begin{aligned}
& \lambda_{\alpha, \mathcal{A}}(\mid \psi>)=\left(\sigma_{x}, \sigma_{z}\right)(\mid \psi>)= \\
= & {\left[\left(\sigma_{x}(\mid 1>)\right)\left(\sigma_{z}(\mid 0>)\right)+\left(\sigma_{x}(\mid 0>)\right)\left(\sigma_{z}(\mid 1>)\right)\right] / \sqrt{2}=} \\
= & {[\mid 0>) \mid 0>)-|1>| 1>)] / \sqrt{2} }
\end{aligned}
$$

which is a Bell state. 
1.6) For $A=\sigma_{y}, B=\sigma_{z}$, thus with $\mathcal{A}=\left\{\sigma_{y}, \sigma_{z}\right\}$, we have in $H \bigotimes_{+, A} H$

$\lambda_{\alpha, \mathcal{A}}(\mid \psi>)=\left(\sigma_{y}, \sigma_{z}\right)(\mid \psi>)=$

$=\left[\left(\sigma_{y}(\mid 1>)\right)\left(\sigma_{z}(\mid 0>)\right)+\left(\sigma_{y}(\mid 0>)\right)\left(\sigma_{z}(\mid 1>)\right)\right] / \sqrt{2}=$

$=[(-i \mid 0>)) \mid 0>)-(i|1>| 1>)] / \sqrt{2}=-i \mid \varphi>$

where

$|\varphi>=| 0>)) \mid 0>)+|1>| 1>$

is a Bell state.

1.7) For $A=B=H$, thus with $\mathcal{A}=\{H\}$, we have in $H \bigotimes_{+, A} H$

$\lambda_{\alpha, \mathcal{A}}(\mid \psi>)=(H, H)(\mid \psi>)=$

$=[(H(\mid 1>))(H(\mid 0>))+(H(\mid 0>))(H(\mid 1>))] / \sqrt{2}=$

$=[((\mid 0>)-\mid 1>) / \sqrt{2})((\mid 0>)+\mid 1>) / \sqrt{2})+$

$+((\mid 0>)+\mid 1>) / \sqrt{2})((\mid 0>)-\mid 1>) / \sqrt{2})] / \sqrt{2}=$

$=[(\mid 0>)-\mid 1>)(\mid 0>)+\mid 1>)+$

$+(\mid 0>)+\mid 1>)(\mid 0>)-\mid 1>)] /(2 \sqrt{2})=$

$=(\mid 0>) \mid 0>)-\mid 1>) \mid 1>)) \sqrt{2}$

which is a Bell state.

Discussion on this paper was started during the visit of A. Khrennikov to University of Pretoria, November 2010, and completed during the visits of E. Rosinger to Linnaeus University, June and September 2011. This research was supported by the joint project of Swedish and South-African Research Councils, "Non-Archimedean analysis: from fundamentals to applications." 


\section{References}

[1] A. Khrennikov, Ubiquitous Quantum Structure: from Psychology to Finances, Springer, Berlin-Heidelberg-New York, 2010.

[2] M. Asano, M. Ohya, Y. Togawa and A. Khrennikov, I. Basieva, "Quantum-like Representation of Bayesian Inference", in Advances in Quantum Theory, AIP Conf. Proc. 1327, American Institute of Physics, New York, 2011, pp. 57-62.

[3] E. E Rosinger, physics.gen-ph-0701116v8.

[4] A. Khrennikov, Non-Archimedean analysis: quantum paradoxes, dynamical systems and biological models, Kluwer, Dordreht, 1997.

[5] E. E Rosinger, A. Khrennikov, "Beyond Archimedean Space-Time Structure", in Advances in Quantum Theory, AIP Conf. Proc. 1327, American Institute of Physics, New York, 2011, pp. 520-526. 